\title{
Evaluation de la qualité physico-chimique et hygiénique du lait de brousse et des produits laitiers locaux commercialisés dans les bars laitiers de N'Djamena au Tchad
}

\author{
M.O. Koussou ${ }^{1 *}$ P. Grimaud ${ }^{2}$ L.Y. Mopaté ${ }^{1}$
} Mots-clés
Produit laitier - Lait fermenté -
Qualité - Hygiène du lait - Tchad.

\begin{abstract}
Résumé
L'objet de l'étude a été d'évaluer la qualité du lait de brousse, du lait entier et du lait fermenté produits dans les bars laitiers de N'Djamena où la demande en produits laitiers est croissante. Pour cela, 30 échantillons de lait de brousse et 40 de lait fermenté ont été prélevés et soumis à des tests physico-chimiques et microbiologiques. Les résultats des analyses ont montré une meilleure qualité du lait de brousse en saison pluvieuse. Bien que la charge microbienne ait atteint un niveau élevé (> 36,106 UFC/mL) quelle qu'ait été la saison, on pouvait observer que les populations de coliformes totaux et fécaux étaient plus nombreuses dans le lait fermenté entier et en saison froide. Cette étude a révélé des insuffisances dans les pratiques d'hygiène au niveau de la production, de la collecte et des procédés de transformation, mais aussi l'absence de moyens de conservation et l'existence de pratiques de mouillage du lait en saison sèche. Les résultats obtenus suggèrent la nécessité de sensibiliser l'ensemble des acteurs de la filière au respect des bonnes pratiques de production et d'hygiène.
\end{abstract}

\section{INTRODUCTION}

Le Tchad, pays sahélien, possède un cheptel important de ruminants : 6,6 millions de bovins, 9 millions de petits ruminants et 1,2 million de camélidés (18). La production annuelle de lait, toutes espèces confondues, a été estimée à $223500 \mathrm{t}$, soit un disponible de $28 \mathrm{~kg} / \mathrm{hab}$./an (15), très en deçà des recommandations de la FAO pour la consommation de lait $(60 \mathrm{~L} /$ personne/an).

N'Djamena, principale ville du Tchad, comptait 800000 habitants en 2005. Sa population croît à un rythme de 6 p. 100 par an (19). Cette poussée démographique s'accompagne d'une hausse de la demande en lait et produits laitiers, comme dans de nombreux pays africains $(7,17)$.

Le bassin d'approvisionnement de la ville en lait repose sur des élevages situés dans un rayon de $65 \mathrm{~km}$. Il s'agit d'élevages pastoraux et agropastoraux qui commercialisent surtout du lait de vache, mais aussi du lait de chèvre et, depuis quelques années, du

\footnotetext{
1. Laboratoire de recherches vétérinaires et zootechniques, BP 433, N'Djamena, Tchad.

2. Lrvz, N'Djamena, Tchad ; Cirad, UPR Systèmes d'élevage,

F-34398 Montpellier, France.

* Auteur pour la correspondance

Tél. : +2356253425 ; e-mail : koussou59@yahoo.fr
}

lait de chamelle (9). Peu d'éleveurs pratiquent la complémentation alimentaire (15). La production suit les variations de la disponibilité en pâturage au cours des différentes saisons. La saison de forte production laitière est donc la saison de pluie qui va de juin à octobre et pendant laquelle les ressources pastorales sont les plus abondantes.

La filière d'approvisionnement de la ville de N'Djamena en lait local a connu un essor considérable dû à l'apparition et à la multiplication de petites entreprises laitières à partir des années 1990, au développement de la consommation hors foyer de lait entier sucré, ainsi qu'au succès du rayeb, un lait entier fermenté local (9). Ces produits correspondent à un besoin nouveau des populations urbaines pour les boissons rafraîchissantes.

Si le lait est un produit à haute valeur nutritive, sa composition et ses propriétés physico-chimiques en font un milieu très favorable à la multiplication des micro-organismes (11). Au Tchad, le secteur de la transformation laitière est marqué par un faible niveau de technicité des entreprises qui, dans leur quasi-totalité, utilisent le lait de brousse comme matière première. Le problème de la qualité sanitaire du lait et des produits laitiers commercialisés se pose donc pour assurer la sécurité des consommateurs. La présente étude vise à déterminer la qualité du lait de brousse livré par les collecteurs aux petites entreprises de transformation en ville, ainsi que celle des produits issus de la transformation. 


\section{MATERIEL ET METHODES}

Les bars laitiers ayant fait l'objet de l'étude sont de petites entreprises laitières localisées à N'Djamena. Ils produisent et commercialisent deux types de produits laitiers : le lait entier sucré et le lait fermenté entier sucré. Ce dernier est obtenu par fermentation naturelle à la température ambiante dans des récipients. Le lait entier sucré est le lait de brousse auquel on a rajouté du sucre et qui est conservé au frais. Des collecteurs de lait assurent quotidiennement l'approvisionnement en « lait de brousse » (termes qui désignent un lait de grand mélange), bouilli avant d'être commercialisé par les producteurs. Ces laits sont collectés dans des élevages extensifs, situés dans un rayon de $65 \mathrm{~km}$ autour de la ville, puis acheminés en ville par des collecteurs à mobylette, à vélo ou en camion. Dix unités de transformation laitière et dix collecteurs à mobylette ont servi de cadre à la réalisation de cette étude.

Les échantillons de lait de brousse $(100 \mathrm{~mL})$ ont été prélevés le matin à l'arrivée des collecteurs. Ceux de lait entier et de lait fermenté sucré $(100 \mathrm{~mL})$ ont été prélevés après chaque fabrication, à partir du lait de brousse ayant fait l'objet de contrôle. Lorsqu'une unité de transformation fabriquait et commercialisait les deux types de produit (lait entier et lait fermenté) à partir du même lait de brousse, les deux étaient prélevés. Les échantillons prélevés ont été soumis aux analyses de température, de $\mathrm{pH}$, puis ils ont été placés dans une glacière pour être acheminés au laboratoire où ont eu lieu les analyses microbiologiques. Le temps entre les prélèvements et les premières analyses n'a pas dépassé une heure. L'opération a été répétée à chaque saison : froide (de novembre à février), chaude (de mars à mai) et pluvieuse (de juin à octobre). Le tableau I montre le nombre d'échantillons prélevés par type de produits et par saison.

Les analyses physico-chimiques ont consisté à mesurer : la température (avec un thermomètre électronique à sonde) ; le $\mathrm{pH}$ (Hanna instruments), la densité (avec un thermolactodensimètre réglé à une température de $20^{\circ} \mathrm{C}$ ) ; l'acidité Dornic par un mélange de $10 \mathrm{~mL}$ de lait et 2 à 3 gouttes de phénolphtaléine à 1 p. $100(\mathrm{w} / \mathrm{v})$ dans de l'éthanol, puis titration par solution de $\mathrm{NaOH}(\mathrm{N} / 9)$; la matière sèche, après dessiccation par évaporation à $103{ }^{\circ} \mathrm{C}$ pendant $4 \mathrm{~h}$ de $5 \mathrm{~g}$ de lait déposé dans une capsule séchée a $103{ }^{\circ} \mathrm{C}$ pendant $4 \mathrm{~h}$ puis tarée ; les cendres totales, après incinération au four à moufle à $500{ }^{\circ} \mathrm{C}$ pendant $3 \mathrm{~h}$ de $5 \mathrm{~g}$ de lait déposé dans une capsule sèche et tarée ; le taux de matière grasse par la méthode de Gerber ; dans un butyromètre ont été introduits $10 \mathrm{~mL}$ d'acide sulfurique concentré, puis $11 \mathrm{~mL}$ de lait et $1 \mathrm{~mL}$ d'alcool amylique ; le butyromètre a été bouché puis retourné 3 à 4 fois pour bien mélanger les trois produits ; la lecture directe du taux de matière s'est faite sur la branche graduée du butyromètre retourné.

\section{Tableau I}

Répartition des échantillons en fonction du type de produit et de la saison

\begin{tabular}{lcccc} 
& $\begin{array}{c}\text { Saison } \\
\text { chaude }\end{array}$ & $\begin{array}{c}\text { Saison } \\
\text { froide }\end{array}$ & $\begin{array}{c}\text { Saison } \\
\text { pluvieuse }\end{array}$ & Total \\
\hline Lait de brousse & 10 & 10 & 10 & 30 \\
Lait entier sucré & 8 & 8 & 8 & 24 \\
Lait entier fermenté sucré & 4 & 8 & 4 & 16 \\
Total & 23 & 27 & 21 & 70
\end{tabular}

Concernant les analyses microbiologiques, la flore aérobie mésophile totale a été dénombrée sur milieu PCA (plate count agar) en surface, à la suite d'une série de dilution de facteur 10 et après incubation dans l'étuve pendant $24 \mathrm{~h}$ à $30^{\circ} \mathrm{C}$; les coliformes ont été dénombrés sur milieu de MacConkey après incubation pendant $24 \mathrm{~h}$ à $37^{\circ} \mathrm{C}$ pour les coliformes totaux et à $44^{\circ} \mathrm{C}$ pour les coliformes fécaux.

Les analyses statistiques ont été effectuées avec le logiciel Spss 12.0 pour Windows. Le modèle suivant a été testé : $Y=\mu+n_{i}+s_{j}$ $+n_{i} s_{j}+e$, où $Y$ représentait la variable dépendante, $\mu$ la moyenne, $\mathrm{n}$ le type de produit ( $\mathrm{i}=1$ ou 2 pour les analyses des paramètres physico-chimiques, et pour la flore totale, et $\mathrm{i}=1$ à 3 pour les analyses de coliformes totaux et fécaux), s la saison ( $\mathrm{j}=1,2$ ou 3$), \mathrm{n}_{\mathrm{i}} \mathrm{s}_{\mathrm{j}}$ l'interaction, et e l'erreur. Lorsque l'effet saison était significatif, le test de Ryan, Einot, Gabriel et Welsch (R-E-G-W) a été mis en œuvre. Le seuil de signification a été fixé à $\mathrm{P}<0,05$.

\section{RESULTATS}

Les valeurs obtenues en saison pluvieuse ont été différentes de celles obtenues en saison sèche froide et chaude pour les différents facteurs (tableau II) : le $\mathrm{pH}$ a été inférieur $(\mathrm{P}<0,05)$ en saison des pluies ainsi que la température $(\mathrm{P}<0,01)$, alors que les teneurs en matière sèche et en cendres totales ont été supérieures au seuil respectif de 1 p. 1000 et 5 p. 100. Les valeurs de la densité et de l'acidité Dornic ont également été significativement supérieures $(\mathrm{P}<0,001)$ en saison des pluies. Les résultats des analyses microbiologiques ont été très variables, et ce, sans effet saisonnier notable (tableau II).

Les valeurs des dénombrements des coliformes totaux ont été significativement différentes ( $\mathrm{P}<0,05$; tableau III) selon la nature du produit, avec une interaction avec la saison $(\mathrm{P}<0,05)$ : soit des valeurs plus faibles pour le lait de brousse en saison sèche froide, alors qu'il n'y a pas eu de différence entre les saisons chaude et pluvieuse. Un effet saison $(\mathrm{P}<0,05)$ a été observé pour les coliformes fécaux, avec une interaction entre la saison et la nature du produit également significative $(\mathrm{P}<0,05)$, soulignant une moins bonne qualité du lait fermenté entier sucré en saison sèche froide.

\section{DISCUSSION}

Les dénombrements des germes et les tests physico-chimiques ont révélé une qualité insuffisante du lait commercialisé. Les mêmes observations ont été faites au Tchad (6), au Mali $(3,4)$ et en Ouganda (13). L'acidité élevée du lait au cours des différentes saisons renseigne sur la présence de germes. Selon Alais (1), l'acidité de titration est la somme de quatre réactions. Les trois premières représentent l'acidité naturelle du lait (acidité due à la caséine, aux sels minéraux et aux phosphates) et la dernière est liée à l'acidité « développée », due à l'acide lactique et aux autres acides provenant de la dégradation microbienne du lactose et éventuellement des lipides en voie d'altération. Le lait contient peu de micro-organismes lorsqu'il est prélevé dans de bonnes conditions à partir d'un animal sain (11). Pour la flore aérobie mésophile totale, la Communauté d'Afrique de l'Est (EAC) fixe à $10^{6} \mathrm{UFC} / \mathrm{mL}$ la valeur en dessous de laquelle le lait est jugé de bonne qualité microbiologique (10). Cette valeur, indicatrice de bonnes pratiques d'hygiène, a été largement dépassée quelle qu'ait été la saison lorsque le lait était réceptionné par les unités de transformation.

Les populations de coliformes ont été plus nombreuses dans le lait de brousse en saison sèche froide et en saison pluvieuse. Les coliformes sont les hôtes habituels de l'intestin des mammifères ; leur présence dans le lait est l'indice d'une contamination fécale 


\section{Tableau II}

Caractéristiques physico-chimiques et microbiologiques moyennes des laits de brousse suivant la saison

\begin{tabular}{|c|c|c|c|c|c|c|c|}
\hline \multirow[b]{2}{*}{ Analyse } & \multicolumn{6}{|c|}{ Saison } & \multirow[b]{2}{*}{ Signification } \\
\hline & froide & ES & chaude & ES & pluvieuse & ES & \\
\hline Matière sèche (g/L) & $124,9^{\mathrm{a}}$ & 0,4 & $118,3^{\mathrm{a}}$ & 0,3 & $136,7^{b}$ & 0,2 & $* * *$ \\
\hline Matières grasses (g/L) & 45,2 & 0,9 & 47,9 & 0,1 & 46,4 & 0,1 & ns \\
\hline Cendres totales $(\mathrm{g} / \mathrm{L})$ & $5,8^{\mathrm{a}}$ & 0,4 & $5,8^{\mathrm{a}}$ & 0,4 & $7,3^{b}$ & 0,1 & * \\
\hline Température $\left({ }^{\circ} \mathrm{C}\right)$ & $43,30^{\mathrm{a}}$ & 1,09 & $44,90^{\mathrm{a}}$ & 0,91 & $39,90^{\mathrm{b}}$ & 1,13 & $* *$ \\
\hline $\mathrm{pH}$ & $5,9^{\mathrm{a}}$ & 0,11 & $5,9^{a}$ & 0,07 & $5,6 b$ & 0,05 & $*$ \\
\hline Acidité totale (degrés Dornic) & $21,1^{\mathrm{a}}$ & 0,9 & $16,1 b$ & 0,8 & $24,2^{\mathrm{c}}$ & 0,8 & $* * *$ \\
\hline Densité à $20^{\circ} \mathrm{C}$ & $1,023^{\mathrm{a}}$ & 0,001 & $1,025^{b}$ & 0,001 & $1,031^{\mathrm{c}}$ & 0,001 & $* * *$ \\
\hline FAMT (x 106) (UFC/mL) & 28,4 & 17,79 & 38,4 & 8,41 & 46,3 & 7,00 & ns \\
\hline Coliformes totaux (UFC/mL) & 5,1 & 1,90 & 66,2 & 25,4 & 51,1 & 26,89 & ns \\
\hline Coliformes fécaux UFC/mL) & 0,9 & 0,60 & 6,7 & 4,32 & 7,3 & 6,36 & ns \\
\hline
\end{tabular}

ES : erreur standard de la moyenne

Les moyennes suivies de lettres distinctes sur une même ligne sont significativement différentes

$* \mathrm{p}<0,05 ; * * \mathrm{p}<0,01 ; * * * \mathrm{p}<0,001 ; \mathrm{ns}:$ non significatif

\section{Tableau III}

Résultats moyens du dénombrement des coliformes selon le type de produit et la saison

\begin{tabular}{|c|c|c|c|c|c|c|c|c|}
\hline \multirow[b]{2}{*}{ Paramètre } & \multirow[b]{2}{*}{ Type de produit } & \multicolumn{6}{|c|}{ Saison } & \multirow[b]{2}{*}{ Effet } \\
\hline & & froide & ES & chaude & ES & pluvieuse & ES & \\
\hline \multirow{3}{*}{$\begin{array}{l}\text { Coliformes totaux } \\
\text { (UFC/mL) }\end{array}$} & Lait de brousse & 5,10 & 1,90 & 66,20 & 25,40 & 51,10 & 26,88 & \multirow{3}{*}{$\mathrm{N}^{*}, \mathrm{SxN} \mathrm{N}^{* *}$} \\
\hline & Lait entier sucré & 205,87 & 66,94 & 51,37 & 25,07 & 57,12 & 36,38 & \\
\hline & Rayeb & 226,75 & 84,78 & 130,7 & 86,93 & 18,00 & 9,51 & \\
\hline \multirow{3}{*}{$\begin{array}{l}\text { Coliformes fécaux } \\
\text { (UFC/mL) }\end{array}$} & Lait de brousse & 0,90 & 0,60 & 6,70 & 4,32 & 7,30 & 6,36 & \multirow{3}{*}{$N^{*}, S^{*}, S x N^{*}$} \\
\hline & Lait entier sucré & 30,00 & 11,73 & 14,37 & 9,49 & 1,37 & 1,37 & \\
\hline & Rayeb & 11,62 & 5,42 & 2,75 & 1,25 & 7,00 & 4,84 & \\
\hline
\end{tabular}

ES : erreur standard de la moyenne

$\mathrm{N}$ : nature du produit ; $\mathrm{S}$ : saison ; $\mathrm{S} \times \mathrm{N}$ : interaction saison produit ; $* \mathrm{p}<0,05 ; * * \mathrm{p}<0,01$

directe ou indirecte due à des pratiques d'hygiène insuffisantes lors de la traite $(11,12)$. Ce constat confirme les observations de Béchir (2) qui rapporte l'inexistence de pratiques d'hygiène de traite chez les éleveurs dans le bassin de collecte de lait. L'insuffisance de la qualité sanitaire des laits de brousse a été soulignée au Tchad par de nombreux auteurs $(6,8,20)$ ainsi qu'au Mali $(3,5)$, où la flore aérobie mésophile totale et les teneurs en coliformes étaient comparables aux présents résultats. Le chauffage du lait après la traite à des températures proches de $92{ }^{\circ} \mathrm{C}(20)$ par les producteurs diminue considérablement la contamination microbienne des laits. Ces niveaux élevés de population microbienne étaient sans aucun doute le fait de contamination lors de la collecte du lait au cours des nombreux transvasements brutaux dans du matériel mal désinfecté ou mal séché (tasse, bidon), ou à l'occasion de contacts avec des mains sales. De plus, le lait restait à une température ambiante très élevée pendant toute la durée du transport (1 à 2 heures). L'effet conjugué de la température, associé à l'absence de chaîne de froid créent des conditions particulièrement favorables au développement des bactéries $(3,11)$.
L'approvisionnement de N'Djamena en produits laitiers est fortement marqué par la saisonnalité de la production qui s'explique par un environnement agroclimatique sahélien et par des systèmes d'élevage très extensifs. Le lait manque en période chaude alors que la demande est forte (15). Les faibles valeurs de la densité du lait observées en saison sèche ont révélé l'existence de pratique de mouillage du lait par les producteurs pour augmenter les volumes commercialisés. Cette pratique, également observée chez les producteurs de lait dans la zone périurbaine de Niamey au Niger et de Bamako au Mali $(4,21)$, réduit la qualité nutritive du lait, notamment sa teneur en matière sèche et en cendres totales, et augmente les risques de contamination.

La transformation n'a pas amélioré la qualité. Ainsi, le lait entier et le lait fermenté sucré étaient de mauvaise qualité sanitaire et plus particulièrement en saison sèche froide eu égard aux résultats moyens du dénombrement des coliformes. Autant une augmentation des flores microbiennes pouvait être attendue pour le lait sucré par l'usage de sucre de mauvaise qualité microbiologique et des pratiques peu hygiéniques, autant la présence de coliformes 
dans le lait fermenté était inattendue. En effet, la fermentation, si elle conduit à une acidification suffisante $(\mathrm{pH}<4,5)$ du lait, doit interdire la croissance, voire faire disparaître les coliformes dans le lait fermenté car ces bactéries ne sont pas acidophiles. Cependant, des études ont montré que certains coliformes étaient capables de résister à l'acidification quand ils sont présents dès le début de la fermentation avec néanmoins une forte mortalité. Une autre hypothèse serait l'insuffisance de l'acidification lors de la fermentation qui ne conduit pas à des valeurs de $\mathrm{pH}$ suffisamment basses et des concentrations en acide lactique trop faibles. Sans données sur le $\mathrm{pH}$ du lait fermenté, il est difficile de conclure.

On peut toutefois affirmer que la présence de coliformes dans ces produits laitiers témoigne de mauvaises conditions d'hygiène au niveau des procédés de fabrication, et d'un environnement insalubre. Ce problème est amplifié par les conditions climatiques, car la chaleur et l'humidité ambiante ne favorisent pas la conservation du lait. Les micro-entreprises laitières de N'Djamena possèdent, pour 92 p. 100 d'entre elles, un congélateur ou un réfrigérateur en état de marche (9), mais les délestages électriques particulièrement fréquents ne permettent pas leur fonctionnement à plein temps. Les tenanciers des bars laitiers ont souvent recours à de la glace, ce qui ne garantit pas une bonne température de conservation.

La forte contamination du lait entier et du lait fermenté commercialisé à certaines périodes pose un problème majeur de santé publique. Il serait intéressant d'approfondir cette étude en établissant le lien entre les pratiques à risque et la qualité des produits, tout en identifiant les points critiques à maitriser pour s'assurer de la qualité sanitaire de ces produits. Par ailleurs, la qualité des laits serait améliorée par de meilleures pratiques $(2,5)$ concernant : l'hygiène de la traite, les barèmes des traitements thermiques de chauffage du lait à la réception par les unités de transformation, le lavage des ustensiles et des bidons au savon ou à l'eau de Javel avec de l'eau de bonne qualité, la réduction du temps de transport ou l'utilisation de la lactoperoxydase par les producteurs de lait et, enfin, la maitrise de la qualité du sucre et de la conduite des fermentations.

\section{CONCLUSION}

Les résultats des analyses physico-chimiques et microbiologiques ont mis en évidence l'insuffisance de la qualité sanitaire du lait et des produits laitiers locaux commercialisés à N'Djamena. La principale cause en est le manque d'hygiène et de salubrité aux différents maillons de la filière et la non maitrise des procédés de transformation. Les risques sont réels pour les consommateurs en l'absence de réglementations et de structures de contrôle des produits permettant d'assurer une qualité hygiénique suffisante des produits laitiers commercialisés. Un travail de sensibilisation et de formation individuelle ou collective des acteurs de la filière aux règles d'hygiène et aux procédés de transformation est indispensable. L'approche de la gestion de la qualité le long de la filière par les organisations professionnelles des acteurs est à privilégier. La réussite d'une telle démarche passe par l'instauration d'une prime à la qualité. Cette pratique est déjà mise en œuvre par quelques transformateurs locaux.

\section{Remerciements}

La présente étude a été possible grâce à un appui financier du Projet d'appui aux services agricoles et aux organisations de producteurs (Psaop). Nous exprimons nos remerciements à la coordination dudit projet, aux collecteurs de lait et aux unités de transformation pour leur collaboration.

\section{BIBLIOGRAPHIE}

1. ALAIS C., 1984. Science du lait. Principes des techniques laitières. Paris, France, Sepaic, 814 p.

2. BECHIR M., 2006. Amélioration de I'hygiène dans la chaîne de production laitière par utilisation du matériel approprié et une meilleure technique de nettoyage et de désinfection : cas de la laiterie traditionnelle "Total » à N'Djamena (Tchad). Mém. DEA Production animales, Eismv, Dakar Sénégal, $31 \mathrm{p}$.

3. BONFOH B., FANE A., ROTH C., TRAORE N.A., COULIBALY Z., SIMBE C.F., ALFAROUKH O.I., NICOLET J., FARAH Z., ZINSSTAG J., 2002. Qualité microbiologique du lait et des produits laitiers vendus en saison sèche dans le district de Bamako au Mali. Revue inter. Sci. Vie Terre ( $\mathrm{n}^{\circ}$ spécial) : 242-250.

4. BONFOH B., FANE A., DEM S., TRAORE H., SIMBE C.F., ALFAROUKH I., NICOLET J., FARAH Z., ZINSSTAG J., 2004. Caractéristiques physicochimiques et biologiques du lait et des produits laitiers vendus à Bamako. Etud. Rech. sahél., 8-9 : 7-12.

5. BONFOH B., WASEM A., ROTH C., TRAORE A.N., SIMBE C.F., ALFAROUKH I., NICOLET J., FARAH Z., ZINSSTAG J., 2004. Les sources de contamination du lait local et les méthodes d'amélioration de sa qualité microbiologique à Bamako (Mali). Etud. Rech. sahél., 8-9 : 29-37.
6. BORNAREL P., BOULBAYE N., HUGOO P., KOUSSOU M.O., 1995. Etat de la situation sanitaire des produits laitiers commercialisés dans la zone périurbaine de N'Djamena. In : Bourzat D. éd., Actes du comité scientifique du Projet régional de recherche sur les petits ruminants (Prrpr), Niamey, Niger, 7-12 fév. 1994, p. 61-73.

7. DELGADO C., ROSEGRANT M., STEINFELD H., EHUI S., COURBOIS C., 1999. L'élevage d'ici 2020 : la prochaine révolution verte. Rome, Italie, FAO, 43 p. (Série Alimentation, agriculture et environnement n²8)

8. DUCRUET P., 1968. Enquête laitière dans la région de Fort-Lamy. Rapport technique. Rome, Italie, FAO, 19 p.

9. DUTEURTRE G., KOUSSOU M.O., SOULEYMAN E.N'G., 2005. Les « bars laitiers » de N'Djamena (Tchad). Des petites entreprises qui valorisent le lait de brousse. In : Raimond C., Garine E., Langlois O., éds, Actes $11^{\mathrm{e}}$ Coll. int. Ressources vivrières et choix alimentaires dans le bassin du lac Tchad, Université de Paris X, Nanterre, France, 20-22 nov. 2002, p. 435-456.

10. EAST AFRICAN COMMUNITY, 1999. East African standards. Unprocessed (raw) whole milk - Specification. Arusha, Tanzania, EAC, $14 \mathrm{p}$.

11. FAYE B., LOISEAU G., 2002. Sources de contamination dans les filières laitières et exemples de démarches de qualité. In: Hanak E., Boutrif E., Fabre P., Pineiro M., Eds, Proc. int. Workshop, Food safety management in developing countries, Montpellier, France, 11-13 Dec. 2000. Montpellier, France, Cirad, CD-ROM.

12. GUIRAUD J.-P., 1998. Analyse du lait. In : Microbiologie alimentaire. Paris, France, Dunod, p. 387-413. 
13. GRILLET N., GRIMAUD P., LOISEAU G., WESUTA M., FAYE B., 2005. Qualité sanitaire du lait cru tout au long de la filière dans le district de Mbarara et la ville de Kampala en Ouganda. Revue Elev. Méd. vét. Pays trop., $58: 245-255$.

14. KOUSSOU M.O., 2004. Dynamique des innovations dans le secteur de l'élevage au Tchad : le cas de la filière d'approvisionnement de la ville de N'Djamena en lait. Mém. DEA, INA-PG, Paris, France, 44 p.

15. KOUSSOU M.O., MOPATE L.Y., 2006. Le bassin d'approvisionnement de la ville de N'Djamena en lait : zonage et typologie des élevages laitiers. Revue afr. Santé Prod. Anim., 4 : 39-44.

16. LAMBERT J.C., LHOSTE F., 2004. La méthode de conservation du lait cru par le système fondé sur la lactoperoxydase. L'intégration du " programme lait de brousse » au module laitier villageois. Etud. Rech. sahél., 8-9 : 67-72.

17. METZGER R., CENTRES J.M., THOMAS L., LAMBERT J.C., 1995. L'approvisionnement des villes africaines en lait et produits laitiers. Rome, Italie, FAO, 101 p. (Production et santé animale $\mathrm{n}^{\circ} 124$ )
18. MINISTERE DU PLAN ET DE LA COOPERATION, 1995. Recensement général de population et de I'habitat, 1993, vol. 3, tome 2 : Etat de la population. N'Djamena, Tchad, ministère du Plan et de la Coopération, $164 \mathrm{p}$.

19. MINISTERE DE L'ELEVAGE, 2006. Rapport annuel des statistiques 2004 et 2005. N'Djamena, Tchad, ministère de l'Elevage, 79 p.

20. PISSANG D.T., 1995. L'évaluation de la qualité des laits et produits laitiers dans les systèmes traditionnels de transformation au Tchad. Mém. Master Technologie agroalimentaire des régions chaudes, Ensia/ Siarc, Montpellier, France, $62 \mathrm{p}$.

21. SIOUSARRAN V., 2003. L'hygiène du lait cru en zones urbaine et périurbaine de Niamey, Niger. Rapport de stage. DESS Productions animales en régions chaudes, Cirad-emvt, Université de Montpellier II, France, $54 \mathrm{p}$.

Accepté le 15 décembre 2009

\section{Summary}

Koussou M.O., Grimaud P., Mopaté L.Y. Hygienic and Physico-Chemical Quality of Local Milk and Milk Products Sold in Milk Bars in Chad

The objective of this study was to assess the quality of local, whole, and fermented milk produced in the milk bars of N'Djamena, where the demand for dairy products is increasing. Thirty samples from the bush and 40 from cultured milk were collected to undergo physico-chemical and microbiological tests. Analysis of the results showed that the bush milk quality was better during the rainy season. The microbiological load reached a high level $\left(>36.10^{6} \mathrm{UFC} / \mathrm{mL}\right)$ for bush milk regardless of the season, but total and fecal coliforms were found in greater quantities in the fermented whole milk during the cold season. The study revealed insufficient hygiene practices at the levels of production, collection and transformation, as well as the absence of conservation means, and sometimes milk adulteration during the dry season. The results suggest the need to make all stakeholders of this subsector aware of the importance of applying good production and hygiene practices.

Keywords: Milk product - Cultured milk - Quality - Dairy hygiene - Chad.

\section{Sumario}

Koussou M.O., Grimaud P., Mopaté L.Y. Evaluación de la calidad físico-química e higiénica de la "leche de monte" y de los productos lecheros locales comercializados en los bares lecheros de N'Djamena (Chad)

El objetivo del presente estudio fue el de evaluar la calidad de leche de monte, de leche entera y de leche fermentada producidos en los bares lecheros de N'Djamena, en donde la demanda en productos lecheros está en crecimiento. Para esto, se tomaron 30 muestras de leche de monte y 40 de leche fermentada y se sometieron a testes físico-químicos y microbiológicos. Los resultados de los análisis mostraron una mejor calidad de leche de monte durante la estación Iluviosa. Aunque la carga microbiana alcanzó un nivel elevado (> 36,106 UFC/mL) indiferentemente de la estación, se podían observar poblaciones de coliformes totales y fecales más numerosas en la leche fermentada entera y durante la estación fría. Este estudio mostró insuficiencias en las prácticas de higiene a nivel de la producción, la colecta y de los procedimientos de transformación, pero también la ausencia de medios de conservación y la existencia de prácticas de humidificación de la leche durante la estación seca. Los resultados obtenidos sugieren la necesidad de sensibilizar al conjunto de los actores de la filial con respecto a las buenas prácticas de producción y de higiene.

Palabras clave: Producto lácteo - Leche fermentada Calidad - Higiene de la leche - Chad. 